\title{
Acalculous Cholecystitis with Spontaneous Gall Bladder Perforation in a Postoperative Case of Radical Cystectomy with Ileal Conduit
}

\author{
Dr. Priyank Yadav, Dr. Vijay Sharma, Dr. Devarshi Srivastava, Dr. Anil Mandhani \\ Department of Urology and Renal Transplantation, SGPGIMS, Raebareilly Road, Lucknow, India \\ priyankmamc@gmail.com,vj_sharma80@yahoo.com,devarshi.sri@gmail.com, \\ mandhani@sgpgi.ac.in
}

\begin{abstract}
Acalculus cholecystitis is an uncommon cause of postoperative paralytic ileus and usually occurs in severely ill patients. The exact pathophysiology is unknown. Its presentation is usually masked by the underlying severe illness. Complication rates are high compared to calculus cholecystitis. It is rarely reported after urological procedures and has not been reported after radical cystectomy. Early diagnosis and intervention is associated with favourable outcome. The present case focuses on the need for early investigation and management of unsettled abdomen after radical cystectomy with ileal conduit.
\end{abstract}

Keywords: Acalculus cholecystitis, cholecystostomy, gall bladder perforation, ileal conduit, radical cystectomy,

\section{INTRODUCTION}

Acalculus cholecystitis is a rare condition which is usually seen in critically ill patients. The pathophysiology is unknown but probably related to biliary stasis and subsequent gall bladder inflammation. The incidence of gall bladder perforation after acalculus cholecystitis is $50-60 \%$ and the mortality rates after such perforation range from $12-16 \%{ }^{1}$. Early diagnosis and surgery is associated with reduced mortality.

Few cases of acute acalculus cholecystitis with gall bladder perforation have been reported in urology practice and none has been reported after radical cystectomy. This case highlights the importance of early diagnosis and prompt treatment of spontaneous gall bladder perforation after radical cystectomy.

\section{CASE Report}

A 60 years old male with history of hypertension and tobacco chewing underwent Radical Cystectomy and Ileal Conduit for muscle invasive transitional cell carcinoma (Fig. 1).

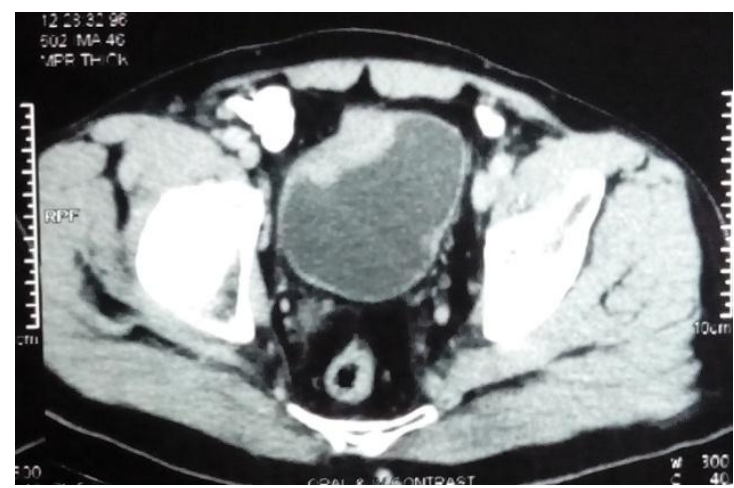

Fig1. Preoperative Contrast Enhanced CT scan Showing an Enhancing Growth at Right Anterolateral Wall of the Urinary Bladder.

Intraoperative course was uneventful. The patient developed abdominal distension and vomiting on postoperative day 2. He did not have any abdominal pain but had low grade fever. Bowel sounds were absent. A nasogastric tube was inserted which drained bilious content. The patient had persistent ileus and was managed conservatively with constant monitoring, fluid and electrolyte replacement. 
On postoperative day 10, CECT abdomen and pelvis was done with oral and IV contrast. It revealed a distended gall bladder with thick and enhancing walls and a perforation at the posterior aspect of the body which was contained by adjacent bowel and omentum(Fig. 2).

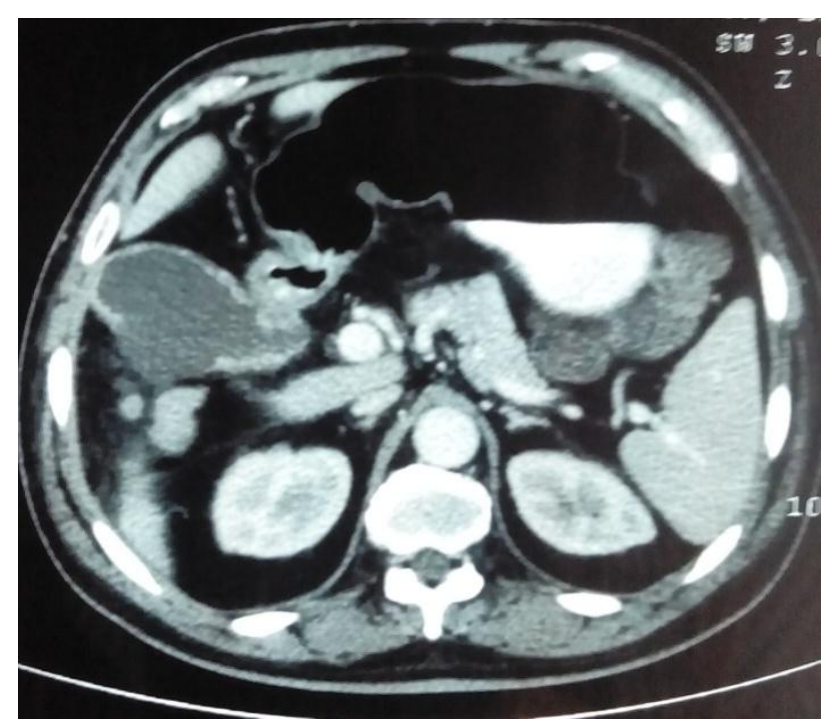

Fig2. Postoperative Contrast Enhanced CT scan Showing Perforation in the Posterior Wall of Body of Gall Bladder.

A percutaneous cholecystostomy was done by the intervention radiologist. Daily output from the cholecystostomy was 800-1000 mL. The paralytic ileus resolved and the patient improved clinically and was discharged. The percutaneous cholecystostomy tube was removed after 2 weeks (when the output decreased to $<50 \mathrm{~mL}$ per day) after doing a tube cholangiogram to ensure proper drainage. The final histopathology was reported as high grade urothelial carcinoma with superficial half muscle invasion with lymph node metastasis (4/17).

\section{DISCUSSION}

Acalculus cholecystitis is a rare condition that is commonly seen in critically ill patients. Most of these patients have history of severe trauma or severe burn injury and are on total parenteral nutrition or mechanical ventilation. Other predisposing factors for acute acalculus cholecystitis include diabetes, acquired immunodeficiency syndrome conditions, steroids and narcotics. It accounts for 5 to $15 \%$ of all cases of acute cholecystitis and has a male preponderance unlike the calculus cholecystitis $^{2}$.

The exact pathophysiology of acute acalculus cholecystitis is not known but it is likely to be an outcome of a combination of ischemia, biliary stasis and sepsis. It has been suggested that in severely ill patients, the absence of stimulus for gall bladder to contract would lead to stasis and formation of biliary sludge ${ }^{3}$. Atherosclerotic microvascular disease associated with diabetes and hypertension may be an added factor to cause spontaneous perforation ${ }^{2}$.

The clinical presentation of acute acalculus cholecystitis is also variable. While outpatients present with the classical features as seen in calculus cholecystitis, majority of the inpatients are severely ill and the symptoms and signs are often masked by the patient's underlying condition or the interventions used to treat it. Still, the commonest features are fever and right upper abdominal pain. Severe complications such as gangrene, perforation and empyema are also more common in acalculus cholecystitis, largely due to the delay in presentation. The incidence of such complications is 50$60 \%$. The commonest site of gall bladder perforation is fundus as the cystic artery is an end artery and fundus is most distant compared to the rest of the gall bladder. In the present case, the site of perforation was in the body of the gall bladder which is an unusual site for perforation.

Diagnosis of acalculus cholecystitis is difficult compared to calculus cholecystitis but when suspected, the initial investigation is USG. However, it is operator dependent and has been reported to be highly sensitive by some series ${ }^{5}$ while others report it to be less sensitive ${ }^{6}$. CT is more sensitive and specific than USG. 
The definitive treatment of acute acalculus cholecystitis is cholecystectomy but this is rarely possible in severely ill patients. A more common approach is percutaneous cholecystostomy tube for decompression of the gall bladder. This may be definitive in itself as there is no chronic obstruction of the gall bladder unlike acute calculus cholecystitis.

The incidence of spontaneous gall bladder perforation after radical cystectomy is not known. Hayes et $\mathrm{al}^{7}$ reported a case of calculus cholecystitis with spontaneous gall bladder perforation with biliary peritonitis 2 weeks after radical cystoprostatectomy. He was found to have ruptured gall bladder with intraperitoneal bile spillage and underwent cholecystectomy with intraperitoneal lavage. In our case, the patient developed acalculus cholecystitis and was managed with a cholecystostomy alone.

To conclude, acalculus cholecystitis is a rare complication seen in surgical practice and is even rarer in urological conditions. Unsettled abdomen after radical cystectomy and the use of ileal segment for diversion should warrant an abdominal CT scan, which was delayed in this patient. Percutaneous cholecystostomy could be a minimally invasive approach to treat spontaneous gall bladder perforation.

ACKNOWLEDGEMENTS - None

CONFLICTS OF INTEREST - No conflicts of interest

\section{REFERENCES}

[1] Lennon F, Green WE. Perforation of the gall bladder. A review of 32 cases. J R Coll Surg Edinb 1983;28:169-73

[2] Ryu JK, Ryu KH, Kim KH. Clinical features of acute acalculous cholecystitis. J Clin Gastroenterol. 2003;36(2):166-9.

[3] Hakala T, Nuutinen PJ, Ruokonen ET, Alhava E. Microangiopathy in acute acalculous cholecystitis. Br J Surg. 1997;84(9):1249-52.

[4] Wang AJ, Wang TE, Lin CC, Lin SC, Shih SC. Clinical predictors of severe gallbladder complications in acute acalculous cholecystitis. World J Gastroenterol. 2003;9(12):2821-3.

[5] Pelinka LE, Schmidhammer R, Hamid L, Mauritz W, Redl H. Acute acalculous cholecystitis after trauma: a prospective study. J Trauma. 2003;55(2):323-9.

[6] Kalliafas S, Ziegler DW, Flancbaum L, Choban PS. Acute acalculous cholecystitis: incidence, risk factors, diagnosis, and outcome. Am Surg. 1998;64(5):471-5.

[7] Hayes DH, Hananel A, Rutchik S, Shirvani A. Spontaneous perforation of the gallbladder as a complication of radical cystoprostatectomy. J Urol. 1999;162(5):1690. 